Posterior ischaemic optic neuropathy after a spontaneous extradural haematoma

Most extradural haematomas (EDH) occur after head injury but cases of "spontaneous" EDH have been reported. ${ }^{12}$ These are a result of either local sepsis, a dural arteriorvenous malformation or an abnormality of clotting. ${ }^{2}$ We report the case of a spontaneous EDH resulting in posterior ischaemic optic neuropathy as a consequence of tentorial herniation.

A 15 year old boy developed a headache which built up over a period of 30 minutes. In the evening he was seen by his general practitioner who diagnosed a viral infection as the headache was associated with some fever. On the second day he vomited in the morning. By the morning of the third day he had fever and was unable to get up because of headache and giddiness. His mother saw him at approximately $1.00 \mathrm{am}$ on the fourth day of his illness when he seemed to be sleeping normally. By 7.00 am the same morning he was found unconscious and was admitted to the District General Hospital on 17 March 1988. There was no other predisposing illness or head injury.

In the accident and emergency department, he was noted to be cyanosed with vomit in his nose and throat. He was in coma with a Glasgow Coma Score of 5. Both pupils did not respond to light and the right was $6 \mathrm{~mm}$, the left $3 \mathrm{~mm}$. His axillary temperature was $40^{\circ} \mathrm{C}$, a regular pulse of 110 , blood pressure $120 / 55 \mathrm{mmHg}$ and normal heart sounds. Auscultation of his chest revealed added sounds in the left base suggesting an aspiration pneumonia which was confirmed on chest $x$ ray. His blood results were as follows: $\mathrm{Hb} 1.58 \mathrm{~g} / \mathrm{l}$, white cell count $11.7 \times 10^{9} / \mathrm{l}$, platelet count $180 \times 10^{9} / 1$, sodium $140 \mathrm{mmol} / \mathrm{l}$, potassium $4.2 \mathrm{mmol} / \mathrm{l}$ and urea $4.1 \mathrm{mmol} / \mathrm{l}$. Clotting was within normal limits with an INR 1.0 and KCCT (control 48).

Before being transferred, the patient was intubated, ventilated, paralysed, sedated and given $100 \mathrm{ml}$ mannitol $20 \%$. On arrival in the neurosurgical unit on 17 March 1988, a CT

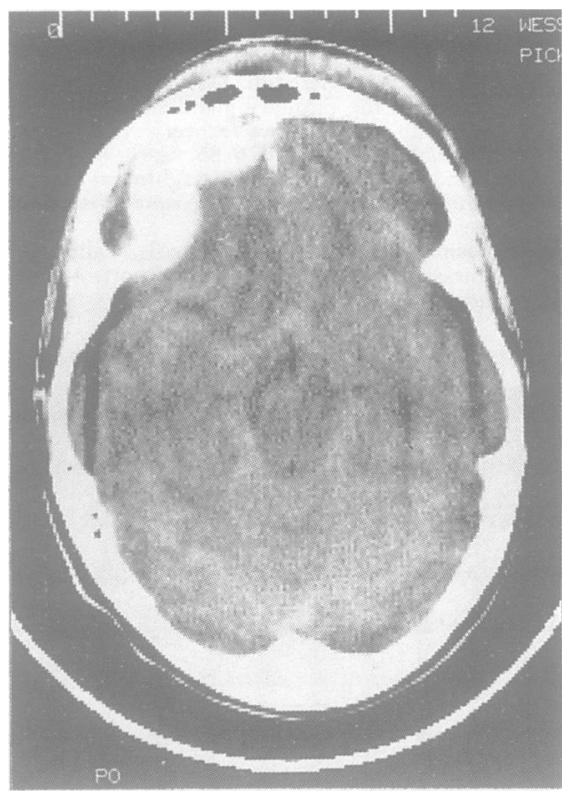

Figure CT scan showing a $3 \mathrm{~cm}$ extradural haematoma of mixed density in the right frontal region. was performed (figure) which showed a $3 \mathrm{~cm}$ maximum depth EDH over the right frontal convexity which was of mixed density. There was no radiological evidence of head injury. In view of his condition and the radiological findings, an immediate right frontal craniotomy was performed at which a moderately sized EDH was found. The haematoma was considered to be of some days duration. No evidence was found of either a fracture or a dural arteriorvenous malformation.

On the evening of the operation the patient developed a transient diuresis which resolved and there were no further episodes. The patient was extubated four days postoperatively and his conscious level continued to improve. Initially his vision was very restricted and he could see only a few objects. Examination showed that the pupils, although unequal, were reacting to light. He appeared to have a left homonymous hemianopia very early after surgery. This was not demonstrable subsequently as he has remained completely blind since then. He was also noted to have a mild left hemiparesis (Medical Research Council grade 4). One month later he was discharged and his conscious level had returned to normal, his hemiparesis had resolved but he was blind.

On review in the outpatient department three months later, his vision was unchanged with no light perception in either eye. He had put on $19 \mathrm{~kg}$ in weight. He had polydipsia and, according to his mother, prolonged and excessive periods of sleeping. On examination he was alert and orientated with no limb weakness. His pupils did not react to light and funduscopy revealed bilateral optic atrophy. Visual evoked responses showed no response. A CT scan showed moderate atrophy of the right frontal lobe and mild dilatation of the ventricular system compared with the previous scan. The orbits, sellae and suprasellar cisterns appeared normal. MRI showed slender optic nerves consistent with atrophy and confirmed the frontal atrophy. there was no evidence of hypothalamic abnormality on the scan. He had an endocrine assessment six months postoperatively which showed an isolated defect of growth hormone production.

One year postoperatively the patient remains blind with fixed pupils. The previous excess of sleeping has resolved and there is no longer excessive thirst. His appetite remains normal and there has been no change in his personality.

The cause of the pregeniculate damage shown by the optic atrophy is not well known, though it is felt to be due to compression of the optic nerves against the skull base by the overlying brain. ${ }^{34}$ This has been shown radiologically in the case of a patient with a falx meningioma where the visual disturbance was reversed postoperatively. ${ }^{5}$ It had not, however, been confirmed pathologically.

In our patient, the CT scan on presentation showed evidence of raised intracranial pressure with obliteration of the basal cisterns and an absent third ventricle. There was no evidence of occipital infarction radiologically on a later scan. We suggest that the cause of this atrophy is a result of infarction secondary to raised intracranial pressure. His visual deterioration, unusually, appeared to have been progressive.

In this patient there was a suggestion of an endocrinopathy with an initial diuresis, polydipsia, weight gain and somnolence. This was, however, not borne out by endocrine assessment which at six months showed an isolated defect in growth hormone production. This finding is of unknown clinical significance but must be recent as the patient is of normal height.

We believe that the cause of the haematoma was truly spontaneous. There was no evidence of other causative factors such as a dural arteriovenous malformation or sepsis at surgery. There was evidence of normal blood coagulation on admission and no history of recent head injury was found after very careful retrospective inquiry.

We would recommend a new classification of EDHs which recognises their cause. Primary EDHs are those haematomas where no cause can be elucidated and secondary EDHs where a cause is demonstrated. CJ GERBER G NEIL-DWYER P KENNEDY Wessex Neurological Centre Southampton General Hospital, Tremona Road, Southampton, UK

Correspondence to: Dr Gerber.

1 Marks SM, Shaw MDM. Spontaneous intracranial extradural haematoma: case report. $f$ Neurosurgery 1982;57:708-9.

2 Sanchis JF, Orozco M, Cabanes J. Spontaneous extradural haematomas. $\mathcal{f}$ Neurol Neurosurg Psychiatry 1975;38:577-80

3 Skarf B, Davis RL, Bisner HA, Hoyt WF Intracranial optic nerve infarction from subdural haematoma. Clinical and pathological findings. Arch Neurol 1984;41:58-60.

4 Keane JR. Blindness following tentorial herniation. Ann Neurol 1980;8:186-90.

5 Klingele TG, Gado MH, Burde RM, Coxe WS Compression of the anterior visual system by the gyrus rectus: case report. $₹$ Neurosurg $1981 ; 55: 272-5$.

A pilot study of N-Methyl-D-Aspartate (NMDA) antagonist in Parkinson's disease

Recent experimental studies have suggested that agents which antagonise glutamatergic transmission could exert anti-Parkinsonian activity. ${ }^{1}$ For example, it has been shown that NMDA antagonists potentiate the effects of levodopa ${ }^{2}$ and could protect substantia nigra from $\mathrm{MPP}^{+}$neurotoxicity ${ }^{3}$ in rats. Injection of the NMDA antagonist MK 801 within the medial pallidum reverses Parkinsonian symptoms in MPTP-treated monkeys. ${ }^{4}$ The mechanism of action remains unclear but it has been suggested that blockade of NMDA receptors would result in a facilitation of dopamine action by preventing the glutamate-induced dephosphorylation of DARPP-32, a dopamine- and cAMP-regulated phosphoprotein. ${ }^{1}$ However, no clinical data are yet available in Parkinson's disease (PD).

We investigated in PD patients the effects of an add-on therapy with ifenprodil, a non competitive antagonist of the NMDA receptor at the polyamine modulatory site, ${ }^{56}$ which inhibits $[3 \mathrm{H}] \mathrm{MK} 801$ binding. ${ }^{7}$ This drug is, as far as we know, the sole NMDA antagonist currently available on the market. Ifenprodil tso possesses alpha-adrenoceptor blocking properties and is already in clinical use as a cerebral anti-ischaemic agent. ${ }^{8}$ 9

Two groups of patients with idiopathic PD were studied after informed consent: the first one included nine non-fluctuating patients mean age (SD), 67 (1) years, mean (SD) duration of PD 9 (1) years, mean (SD) duration of levodopa therapy : 7 (1) years, 
mean (SD) dose of levodopa : 595 (88) mg, mean (SD) stage of Hoehn and Yahr : 3.2 $(0 \cdot 3)$ with waning efficacy of levodopa and who were requiring an increase in PD treatment. The second group included 11 other patients, mean age, 61 (2) years, mean (SD) duration of PD : 12 (1) years, mean (SD) dose of levodopa : 857 (92) $\mathrm{mg}$, mean (SD) duration of levodopa therapy : 10 (1) years, mean (SD) stage of Hoehn and Yahr : 3.0 $(0 \cdot 3)$ with levodopa-induced peak-dose dyskinesias most of the day. In both groups, ifenprodil (Vadilex), (20 mg three times daily orally, the dose currently used in clinical practice) was added in an open design without changing the previous usual treatment. These two kinds of PD patients were selected to investigate if the NMDA antagonist could improve Parkinsonian symptoms (group 1) or modify dyskinesias (group 2). Parkinsonian symptoms were evaluated in the morning (10 am) according to the Unified Parkinson's Disease Rating Scale (UPDRS) before and after 1 month of treatment. Each new assessment was made blind, without the previous scale score in front of the physician. Patients of group 2 were assessed in the "on" condition.

Add-on therapy with ifenprodil did not modify the Parkinsonian symptoms in any group. The total UPDRS motor examination score did not change significantly in group 1 $18.6(3.5)$ vs $20.2(3.2)$ or group 2 [9.4 (2.3) vs $8.6(2.4)]$. The UPDRS subscores for cardinal extrapyramidal symptoms (tremor, rigidity, bradykinesia) or for daily activities did not change (data not shown). The dyskinesia score remained unchanged in group 2 . Side effects were palpitations and sedation ( 1 patient) and feeling of nasal congestion (1 patient).

Our study is the first to investigate the clinical effects of an NMDA antagonist in the treatment of PD. It failed to demonstrate any relevant anti-Parkinsonian effect of ifenprodil. These negative results must, however, be considered with caution. We used the daily dose of ifenprodil recommended for the treatment of intermittent claudication but the pharmacokinetic profile of the drug is poorly known. No published data are available about its plasma half life and brain distribution. It is thus possible that other NMDA antagonists with a better pharmacokinetic profile may exert more potent effects in PD. Since the polyamine modulatory site is only a part of the NMDA receptor complex, ${ }^{67}$ it is also difficult to give a precise biochemical interpretation of our result. Our work does not exclude a definite role for NMDA antagonists in PD. Further studies should be conducted with other NMDA antagonists when available in humans. IL MONTASTRUC O RASCOL JM SENARD A RASCOL * Departments of Medical and Clinical Pharmacology and Neurology, *

Centre Hospitalier Universitaire Purpan, Faculté de Médecine, 37 allées frules-Guesde,
31073 Toulouse, France.

1 Girault JA, Halpain S, Greengard P. Excitatory amino acid antagonists and Parkinson's disease. Trends Neurosci 1990;13:325-6.

2 Klockgether T, Turski L. NMDA antagonists potentiate antiparkinsonian actions of L-Dopa in monoamine-depleted rats. Ann Neuro 1990;28:539-46.

3 Turski L, Bressler K, Rettig KJ, Löschmann PA Wachtel $\mathbf{H}_{\text {. Protection of substantia nigra }}$ from MPP ${ }^{+}$neurotoxicity by N-methyl-D-as4 Graham WC, Robertson RG, Sambrook MA,
Crossman AR. Injection of excitatory amino acid antagonists into the medial pallidal segment of a 1-methyl-4-phenyl-1,2,3,6-tetrahydropyridine (MPTP) treated primate reverses motor symptoms of Parkinsonism. Life Sci 1990;47:92-97.

5 Carter C, Benavides J, Legendre $P$, et al. Ifenprodil and SL 82.0715 as cerebral antiischemic agents. II. Evidence for $\mathrm{N}$-methylD-aspartate receptor antagonist properties. $\mathcal{f}$ Pharmacol Exp Ther 1988;247:1222-32.

6 Carter CJ, Lloyd KG, Zivkivic B, Scatton B. Ifenprodil and SL 82.0715 as cerebral antiischemic agents. III. Evidence for antagonisic effects at the polyamine modulatory site within the N-methnyl-D-aspartate receptor complex. F Pharmacol Exp Ther 1990; 253:475-82

7 Reynolds IJ, Miller RJ. Ifenprodil is a novel type of N-methyl-D-aspartate receptor antagonist: interaction with polyamines. Mol Pharmacol 1989;36:758-65.

8 Happert JL, Catel Ph, Pilardeau P, Loisel JC. Emploi du Vadilex dans le traitement des insuffisants vasculaires cérébraux. Vie Med 1976;57:2334-6.

9 Otomo E, Atarashi J, Azaki G, Ito E, Omae T, Kuzuka F, Nukuda T, Ebi O. Comparison of vinpocetine with ifenprodil tartrate and dihyinocetine with ifenprodil tartrate and dihydroergotoxine mesylate treatment and results of long-term treatment with vinpocetine. Curr
Ther Res 1985;37:811-21.

\section{with AIDS}

Central pontine myelinolysis (CPM) has been associated with rapid correction of hyponatraemia ${ }^{1}$ as well as an acute rise in serum sodium even from normonatraemic levels. ${ }^{2}$ CPM has also been linked with hyperosmolality. ${ }^{3}$ The rate of change in the osmalality, the length of time that this rate of change persists, and the clinical condition of the patient are all important factors. ${ }^{4} \mathrm{We}$ describe a case of CPM in a 49 year old HIV positive homosexual man who had a normal serum sodium and osmolality throughout his illness.

The patient had been HIV positive for at least six years. In 1989 he developed increasing hepatosplenomegaly and uncontrollable thrombocytopenia. A splenectomy was performed and histology showed only nonspecific changes associated with HIV. After an initial stormy postoperative course, he made an excellent recovery and returned to work.

Seven months later he presented with a short history of malaise, anorexia, and fevers. The only abnormal finding was a pericardia rub. Investigations showed that he was mildly anaemic, and hypoalbuminaemic with a serum albumin of $19 \mathrm{~g} /$. Liver function tests and clotting studies were normal. Initial investigations were all negative apart from demonstrating a pericardial effusion and a small pleural effusion. On rescreening for pericardiocentesis, however, the effusion had resolved. A small pleural effusion was aspirated and found to contain numerous lymphocytes suggesting a lymphoma. Immunocytochemistry was unhelpful. Bone marrow examination and $24 \mathrm{~h}$ urine protein excretion were normal. He had no gastrointestinal symptoms. Thirteen days before his death he developed diplopia and was found to have a right sixth nerve palsy. A CT brain scan showed no abnormalities. A lumbar puncture showed clear CSF with 10 lymphocytes and a protein of $0.78 \mathrm{~g} /$. No pathogens were isolated and he was cryptococcal antigen negative. Cytology of the CSF was normal. A trial of high dose steroids made no difference to his deterioration. He became progressively

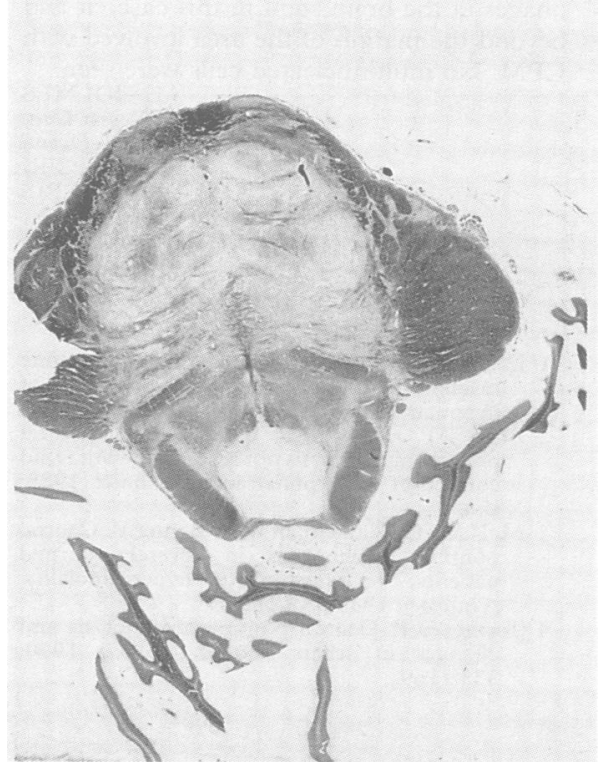

Figure Myelin stained section of the pons showing the area of circumscribed pallor in the base characteristic of CPM.

weaker, developed right-sided ptosis and dysarthria and died a few days later.

Necropsy examination demonstrated disseminated lymphoma, including large ulcerating deposits in the stomach. Postmortem examination of the brain showed the typical appearance of CPM with a large well defined focus of myelin loss in the base of the midpons and upper-pons (figure). Focal infiltrates of lipid containing macrophages were present in the area of myelinolysis but there was no inflammation otherwise associated with this lesion. Axons were partly preserved. There was some mild cerebral atrophy, mild diffuse myelin pallor in the cerebrum and slight lymphomatous infiltration of the meninges.

To out knowledge there have been no previous reports of CPM in association with HIV. The only significant biochemical abnormality was the hypoalbuminaemia which developed in the month preceding his death. This may have been due to gastro-intestinal losses as well as to his underlying condition. His serum sodium remained normal throughout his stay in hospital, and he received no intravenous fluids. His albumin remained at $19 \mathrm{~g} / 1$ until he died. CPM has been described in liver transplant recipients and in servere burns cases, as well as in association with alcoholism and malnutrition. It has been thought that the rapid correction, or over correction, of hyponatraemia was the crucial factor in causing CPM in most cases. This is obviously not the only cause. In a series of CPM in severely burnt patients, hyponatraemia was not a feature, whereas each patient who developed CPM had had a prolonged episode of extreme hyperosmolality. Our patient did not have an episode of serum hyperosmolality, but had one month of hypoalbuminaemia. A low serum albumin may be a significant factor in the development of CPM. This could be a feature common to our patient and the groups of patients described above in which CPM had been reported. Another possibility is that CPM may be a primary effect of HIV, representing yet another neuropathological manifestation of this virus The p24 HIV protein was detected immunocytochemically in rare perivascular macro- 